TRANSACTIONS OF THE

AMERICAN MATHEMATICAL SOCIETY

Volume 361, Number 12, December 2009, Pages 6567-6582

S 0002-9947(09)04754-0

Article electronically published on July 17, 2009

\title{
A SPECTRAL MULTIPLIER THEOREM FOR NON-SELF-ADJOINT OPERATORS
}

\author{
EL MAATI OUHABAZ
}

\begin{abstract}
We prove a spectral multiplier theorem for non-self-adjoint operators. More precisely, we consider non-self-adjoint operators $A: D(A) \subset L^{2} \rightarrow$ $L^{2}$ having numerical range in a sector $\Sigma(w)$ of angle $w$, and whose heat kernel satisfies a Gaussian upper bound. We prove that for every bounded holomorphic function $f$ on $\Sigma(w), f(A)$ acts on $L^{p}$ with $L^{p}$-norm estimated by the behavior of a finite number of derivatives of $f$ on the boundary of $\Sigma(w)$.
\end{abstract}

\section{Introduction}

Let $(X, \mu, \rho)$ be a metric measured space. We denote by $v(x, r)$ the volume of the ball of center $x$ and radius $r$; that is,

$$
v(x, r):=\mu(B(x, r)):=\mu(\{y \in X, \rho(x, y)<r\}) .
$$

We assume that $v(x, r)<\infty$ for all $x \in X$ and $r>0$ and that $X$ satisfies the doubling condition

$$
v(x, 2 r) \leq C_{0} v(x, r) \forall x \in X, r>0,
$$

where $C_{0}$ is a positive constant (independent of $x$ and $r$ ). If (1D) is satisfied, then there exist positive constants $C_{1}$ and $d$ such that

$$
v(x, \lambda r) \leq C_{1} \lambda^{d} v(x, r) \forall x \in X, r>0, \lambda \geq 1 .
$$

Let $\Omega$ be an open subset of $X$. Consider $A: D(A) \subseteq L^{2}(\Omega, \mu) \rightarrow L^{2}(\Omega, \mu)$ a densely defined linear operator. We assume that $A$ is sectorial in the sense that

$$
1 \in \rho(-A) \text { and } W(A) \subseteq \overline{\Sigma(w)} \text {, }
$$

where $W(A)$ is the numerical range of $A, \Sigma(w)=\{z \neq 0,|\arg z|<w\}$, and $w \in[0, \pi / 2)$ is a constant. Under these assumptions, $-A$ generates a strongly continuous semigroup $\left(e^{-t A}\right)_{t \geq 0}$ on $L^{2}(\Omega, \mu)$. Finally, we assume that $e^{-t A}$ is given by a kernel $p(t, x, y)$ (called the heat kernel of $A$ ) which satisfies the global Gaussian upper bound

$$
|p(t, x, y)| \leq \frac{C}{\sqrt{v\left(x, t^{1 / m}\right) v\left(y, t^{1 / m}\right)}} \exp \left\{-c \frac{\rho(x, y)^{m /(m-1)}}{t^{1 /(m-1)}}\right\},
$$

for all $t>0$ and $\mu$-a.e. $x, y \in \Omega$. Here $C, c$ and $m$ are positive constants and $m \geq 2$.

Under conditions (11) and (4), $\left(e^{-t A}\right)_{t \geq 0}$ extends (from $L^{2}(\Omega, \mu) \cap L^{p}(\Omega, \mu)$ ) to a strongly continuous semigroup on $L^{p}(\Omega, \mu)$ for all $p$ with $1 \leq p<\infty$. We keep the same notation and denote by $-A$ the corresponding generator on $L^{p}(\Omega, \mu)$. One of

Received by the editors June 28, 2007 and, in revised form, January 30, 2008.

2000 Mathematics Subject Classification. Primary 42B15; Secondary 47F05.

(C)2009 American Mathematical Society 6567 
the aims of this article is to study the functional calculus $f(A)$ on $L^{p}(\Omega, \mu)$. That is, we look for suitable conditions on a given function $f$ such that $f(A)$ defines a bounded linear operator on $L^{p}(\Omega, \mu)$. We start by recalling some known results.

Assume first that $A$ is self-adjoint (i.e., $w=0$ in (3) ). In that case, $f(A)$ is bounded on $L^{2}(\Omega, \mu)$ for every bounded measurable function $f:[0, \infty) \rightarrow \mathbb{C}$. It is proved in [13] (see also [1, 17] and [24] for related results) that under the assumptions (2) and (4), if $f$ satisfies the condition 1

$$
\sup _{t>0}\|\varphi(.) f(t .)\|_{C^{s}}<\infty, \text { for some } s>d / 2
$$

(and some non-trivial function $\left.\varphi \in C_{c}^{\infty}(0, \infty)\right)$, then $f(A)$ is of weak-type $(1,1)$ and hence bounded on $L^{p}(\Omega, \mu)$ for all $p \in(1, \infty)$.

For non-self-adjoint sectorial operators, one may define the holomorphic functional calculus (see [23, 77). It is proved in 23] that if $A$ satisfies (3), then $f(A) \in \mathcal{L}\left(L^{2}\right)$ for every $f: \Sigma(w+\varepsilon) \rightarrow \mathbb{C}$ that is bounded and holomorphic (here $\varepsilon>0$ ). If in addition (11), (4) are satisfied, then $A$ has a bounded holomorphic functional calculus on $L^{p}(\Omega, \mu)(1<p<\infty)$. This was proved in [11] and later in [12. Therefore, for every $\varepsilon>0$, there exists a positive constant $C_{\varepsilon}$ such that

$$
\|f(A)\|_{\mathcal{L}\left(L^{p}\right)} \leq C_{\varepsilon} \sup _{z \in \Sigma(w+\varepsilon)}|f(z)|
$$

for all bounded holomorphic functions on the sector $\Sigma(w+\varepsilon)$.

One of the results we shall prove in this paper is the following.

Theorem 1. Assume that $X$ satisfies (21) and let $\Omega$ be any open subset of $X$. Assume that $A: D(A) \subset L^{2}(\Omega, \mu) \rightarrow L^{2}(\Omega, \mu)$ satisfies (3) (with some $w \in$ $(0, \pi / 2))$ and (44). Fix $p \in(1,2)$. If $f: \Sigma(w) \rightarrow \mathbb{C}$ is holomorphic and has $[(d / 2+1)|1-2 / p|]+1$ derivatives on the boundary $\mathbb{R}_{+} e^{ \pm i w}$ (where [.] denotes the integer part) and such that

$$
\sup _{\lambda>0} \lambda^{k}\left|f^{(k)}\left(e^{ \pm i w} \lambda\right)\right|<\infty, k=0,1, \ldots,[(d / 2+1)|1-2 / p|]+1,
$$

then $f(A)$ is a bounded operator on $L^{r}(\Omega, \mu)$ for all $r \in\left[p, \frac{p}{p-1}\right]$.

If $p=1$, then $f(A)$ is weak type $(1,1)$ and bounded on $L^{r}(\Omega, \mu)$ for all $r \in(1, \infty)$.

Note that (7) is a Mikhlin-Hörmander type condition on the two rays $\mathbb{R}_{+} e^{-i w}$ and $\mathbb{R}_{+} e^{i w}$. As an application of this theorem, we obtain that the imaginary powers $A^{i s}$ are bounded on $L^{p}(\Omega, \mu)$ with the estimate

$$
\left\|A^{i s}\right\|_{\mathcal{L}\left(L^{p}\right)} \leq C_{p} e^{w|s|}(1+|s|)^{\alpha_{p}}, \quad s \in \mathbb{R},
$$

where $\alpha_{p}=[(d / 2+1)|1-2 / p|]+1$. This estimate is better than the one obtained by the holomorphic functional calculus (6). The latter gives for all $\varepsilon>0$,

$$
\left\|A^{i s}\right\|_{\mathcal{L}\left(L^{p}\right)} \leq C_{p, \varepsilon} e^{(w+\varepsilon)|s|} .
$$

Actually, the multiplier theorem we prove in Section 4 is slightly more general than Theorem 1 which we quote here for simplicity. We shall give a condition on the function $f$ that is more in the spirit of (5). The bound of the $L^{p}$-norm for imaginary powers is slightly better than what we give in the Introduction.

\footnotetext{
${ }^{1}$ This is a Hörmander type condition. If $A$ is the Laplacian on $\mathbb{R}^{d}$, one can replace the norm of the Lipschitz space $C^{s}$ in (5) by that of the Sobolev space $W^{s, 2}$; see [20]. See also [13] for more recent results and several references.
} 
The results in this paper apply for example to non-self-adjoint uniformly elliptic operators on euclidean domains (with Dirichlet or Neumann boundary conditions) as well as to Schrödinger operators with complex-valued potentials. Let

$$
A=\sum_{k, j=1}^{d} \frac{\partial}{\partial x_{j}}\left(a_{k j} \frac{\partial}{\partial x_{k}}\right)
$$

be a uniformly elliptic operator on $L^{2}(\Omega, d x)$ and subject to Dirichlet boundary conditions. If the coefficients $a_{k j}$ are real-valued, then the heat kernel of $A$ satisfies the Gaussian upper bound:

$$
|p(t, x, y)| \leq C t^{-d / 2} \exp \left\{-c \frac{|x-y|^{2}}{t}\right\}, t>0 .
$$

See [24, Chapter 6 . If $A$ is subject to Neumann boundary conditions and $\Omega$ has the extension property, then (8) holds with an additional term $e^{t}$ in the RHS.

For Schrödinger operators $A=-\Delta+V$ on $L^{2}\left(\mathbb{R}^{d}\right)$ with complex-valued potential $V$, we assume (for simplicity) that the real part $\operatorname{Re} V$ is non-negative and locally integrable, and that the imaginary part $\operatorname{Im} V$ satisfies the quadratic form inequality

$$
\left.\left|\int_{\mathbb{R}^{d}} \operatorname{Im} V\right| u\right|^{2} d x \mid \leq c\left[\int_{\mathbb{R}^{d}}|\nabla u|^{2} d x+\int_{\mathbb{R}^{d}} \operatorname{Re} V|u|^{2} d x\right],
$$

for all $u \in W^{1,2}\left(\mathbb{R}^{d}\right)$ for which the RHS is finite. Here $c$ is a non-negative constant. Under these conditions, the operator $A$ satisfies (3) with $w=\arctan c$. In addition, its heat kernel satisfies a Gaussian upper bound. The latter property follows from the domination property

$$
\left|e^{-t A} f\right| \leq e^{-t(-\Delta+\operatorname{Re} V)}|f| \leq e^{t \Delta}|f|,
$$

for all $t>0$ and all $f \in L^{2}\left(\mathbb{R}^{d}, d x\right)$. See, e.g., [24], Theorem 2.21.

Let us note that the case $p=2$ in Theorem 6 was proved in [9] (see also [8]). In order to prove the theorem we shall first prove sharp estimates for the $L^{p}$-norm of the semigroup with complex time $e^{-z A}, z \in \Sigma(\pi / 2-w)$. This, together with the case $p=2$ and a result from [12] on singular integral operators, allows us to prove that $A$ has a bounded holomorphic functional calculus on $L^{p}(\Omega, \mu)$ with a precise estimate. More precisely, we shall prove that $f(A)$ is bounded on $L^{p}(\Omega, \mu)$ with norm bounded by

$$
\|f(A)\|_{\mathcal{L}\left(L^{p}\right)} \leq C \nu^{-\beta} \sup \{|f(z)|, z \in \Sigma(w+\nu)\}
$$

for all functions $f$ that are bounded and holomorphic on $\Sigma(w+\nu)$. Here $\nu>0$ is any arbitrary positive constant and $\beta$ is a positive constant which depends on $d$. Once (9) is proved, we follow similar arguments as in the proof of Theorem $4.10 \mathrm{in}$ [7. (in which $w=0$ ) and deduce the spectral multiplier theorem. Note that there are similarities between our approach and that of [10, where self-adjoint operators were considered. In the case of non-self-adjoint operators, many new arguments are needed. To the best of our knowledge, this is the first paper where spectral multiplier theorems for non-self-adjoint operators are investigated. The literature in the self-adjoint case is huge, and we make no attempt here to provide a complete list of references. We refer the reader to [13.

We also note that, due to our approach (based on the holomorphic functional calculus), we have one more derivative on the function (on $\mathbb{R}_{+} e^{ \pm i w}$ ) than we obtain from (5) in the self-adjoint case ([13]). We believe that the above theorem holds with 
the condition that (77) is valid for $k=0,1, \ldots,[(d / 2+1)|1-2 / p|]$, but this remains to be proved. Another important question is under which conditions on $\Omega$ and $A$ does one have a multiplier theorem with a condition of type (5) on $(0, \infty) e^{ \pm i w}$, but now with the norm of $W^{s, 2}$ instead of $C^{s}$.

We shall prove several other results. In Section 3, we study Riesz means and $L^{p}$-properties of the boundary semigroup $e^{-t e^{i(\pi / 2-w)} A}$. As we shall explain later, the semigroup $e^{-z A}$ acts on $L^{p}(\Omega, \mu)$ for $z \in \Sigma(\pi / 2-w)$ and $1 \leq p<\infty$. However, the boundary value $e^{-t e^{ \pm i \psi} A}$ does not necessarily act on $L^{p}(\Omega, \mu)$ for $p \neq 2$. We define the Riesz mean operator

$$
I_{\alpha}(t):=t^{-\alpha} \int_{0}^{t}(t-s)^{\alpha-1} e^{-s e^{ \pm i \psi} A} d s, \quad t>0,
$$

where $\psi:=\pi / 2-w$. We prove that for every $\alpha>d\left|\frac{1}{2}-\frac{1}{p}\right|, I_{\alpha}(t) \in \mathcal{L}\left(L^{p}(\Omega)\right)$ with $\left\|I_{\alpha}(t)\right\|_{\mathcal{L}\left(L^{p}\right)} \leq C$ for all $t>0$. We also show that the operators $(I+A)^{-\alpha} e^{-t e^{i \psi} A}$ and $(I+A)^{-\alpha} e^{-t e^{-i \psi} A}$ are bounded on $L^{p}(\Omega, \mu)$ with $L^{p}$-norms bounded by $C(1+$ $t)^{\alpha}$. Both results have been known for self-adjoint operators; see Section 3 for references and more comments.

\section{NOTATION}

We shall use the following notation.

$H^{\infty}(\Sigma(v))$ denotes the space of bounded holomorphic functions on the open sector $\Sigma(v)$. For every $f \in H^{\infty}(\Sigma(v))$ we denote by $\|f\|_{v}$ the supremum of $|f|$ on $\Sigma(v)$.

By $\mathcal{L}(E)$ we denote the space of all bounded linear operators on $E$. Its norm will be denoted by $\|\cdot\|_{\mathcal{L}(E)}$.

For every $\alpha \in \mathbb{R},[\alpha]$ denotes its integer part.

A linear operator $T$ is called weak $(1,1)$ if there exists a positive constant $c$ such that for every $\lambda>0$ and every $f \in L^{1}(\Omega)$, we have

$$
\mu(\{x \in \Omega,|T f(x)|>\lambda\}) \leq c \frac{\|f\|_{1}}{\lambda} .
$$

The smallest possible constant $c$ will be denoted $\|T\|_{L^{1}, L^{1, w}}$. All inessential constants will be denoted $C, C^{\prime}, C_{1}, C_{2}, \ldots$.

\section{2. $L^{p}$-ESTIMATES FOR $e^{-z A}$}

Let $A: D(A) \subseteq L^{2}(\Omega, \mu) \rightarrow L^{2}(\Omega, \mu)$ be a densely defined linear operator. We assume that $A$ is sectorial, i.e., $A$ satisfies (3) with some constant $w \in[0, \pi / 2)$. It is well known that under these conditions, $-A$ generates on $L^{2}(\Omega, \mu)$ a holomorphic semigroup $e^{-z A}$, where $z \in \Sigma(\pi / 2-w)$. Moreover,

$$
\left\|e^{-z A}\right\|_{\mathcal{L}\left(L^{2}\right)} \leq 1 \text { for all } z \in \Sigma(\pi / 2-w) .
$$

See, e.g., 21] Theorem 1.24 (p. 492) and 24, Theorem 1.53. In addition, (10) implies that $e^{-z A}$ extends to a strongly continuous semigroup on the boundary $z=t e^{i(\pi / 2-w)}, t \geq 0$ (see Theorems 17.9.1 and 17.9.2 in [19]). The same also holds for $z=t e^{-i(\pi / 2-w)}$. In particular,

$$
\left\|e^{-t e^{ \pm i(\pi / 2-w)} A}\right\|_{\mathcal{L}\left(L^{2}\right)} \leq 1 \text { for all } t \geq 0 .
$$


Assume now that $e^{-t A}$ is given by a kernel $p(t, x, y)$ which satisfies (4). Assume also that $X$ satisfies (11). Under these conditions, the semigroup $\left(e^{-t A}\right)_{t \geq 0}$ extends to all $L^{p}$ spaces and is a holomorphic semigroup on $L^{p}(\Omega, \mu)$ for all $p, 1 \leq p<\infty$, with the same angle $\pi / 2-w$ as on $L^{2}(\Omega, \mu)$ (see Corollary 3 below and the comments at the end of this section).

Our aim now is to prove a sharp estimate for the $L^{p}-$ norm of $e^{-z A}$. This will be a very useful tool in the the proof of the multiplier theorem. Note that the results of this section are known in the case of self-adjoint operators (see [5] or [24]).

We start with the following estimate of the kernel $p(z, x, y)$ of $e^{-z A}$.

Theorem 2. Under the assumptions (11), (3) and (4) we have

$$
\begin{aligned}
|p(z, x, y)| & \leq \frac{C^{\prime}}{\left[v\left(x,\left(\frac{|z| \cos (w+|\theta|)}{\cos w}\right)^{1 / m}\right) v\left(y,\left(\frac{|z| \cos (w+|\theta|)}{\cos w}\right)^{1 / m}\right)\right]^{1 / 2}} \\
& \times \exp \left[-c^{\prime} \frac{\rho(x, y)^{m /(m-1)}}{|z|^{1 /(m-1)}} \cos (w+|\theta|)\right]
\end{aligned}
$$

for all $z \in \Sigma(\pi / 2-w)$. Here $\theta=\arg z$ and $C^{\prime}, c^{\prime}$ are positive constants.

Proof. This result is the non-symmetric version of Theorem 7.3 in 24. Some arguments of the proof are similar to those in [24], and so we shall not give full details.

1) First, the following estimate follows easily from the doubling condition (11) (or equivalently (2) ) (see, e.g., [24, p. 197):

$$
\int_{\Omega} \frac{1}{\left[v\left(x, t^{1 / m}\right) v\left(y, t^{1 / m}\right)\right]^{1 / 2}} \exp \left[-c \frac{\rho(x, y)^{m /(m-1)}}{t^{1 /(m-1)}}\right] d \mu(y) \leq C \text { (a.e.) } x \in \Omega,
$$

where $C$ is a positive constant independent of $t$.

2) We prove that for some constant $C>0$ we have for all $z \in \Sigma(\pi / 2-w)$,

$$
\int_{\Omega}|p(z, x, y)|^{2} d \mu(y) \leq \frac{C}{v\left(x,\left(\frac{|z| \cos (w+|\theta|)}{\cos w}\right)^{1 / m}\right)}
$$

If $\theta:=\arg z \geq 0$, we can write

$$
z=\frac{\operatorname{Re}\left(z e^{i w}\right)}{\cos w}+\frac{\operatorname{Im} z}{\cos w} e^{i(\pi / 2-w)}=: z_{1}+z_{2} .
$$

Therefore, by (11) and the formula $p(z, x, y)=e^{-z_{2} A} p\left(z_{1}, x,.\right)(y)$ (which follows from the semigroup property), one has

$$
\begin{aligned}
\int_{\Omega}|p(z, x, y)|^{2} d \mu(y) & =\left\|e^{-z_{2} A} p\left(z_{1}, x, .\right)\right\|_{2}^{2} \\
& \leq\left\|p\left(z_{1}, x, .\right)\right\|_{2}^{2}
\end{aligned}
$$

Since $z_{1}>0$ we use the Gaussian bound (4) for $p\left(z_{1}, x, y\right)$ and easily estimate the latter term by the RHS of (13) by using (12).

If $\theta<0$, we write $z=\frac{\operatorname{Re}\left(z e^{-i w}\right)}{\cos w}-\frac{\operatorname{Im} z}{\cos w} e^{-i(\pi / 2-w)}$ and argue as above. 
3) We have for all $z \in \Sigma(\pi / 2-w)$

$$
|p(z, x, y)| \leq \frac{C}{\left[v\left(x,\left(\frac{|z| \cos (w+|\theta|)}{\cos w}\right)^{1 / m}\right) v\left(y,\left(\frac{|z| \cos (w+|\theta|)}{\cos w}\right)^{1 / m}\right)\right]^{1 / 2}} .
$$

By the semigroup property, we have $p(z, x, y)=\int_{\Omega} p(z / 2, x, u) p(z / 2, u, y) d \mu(u)$. The desired inequality follows from the Cauchy-Schwarz inequality

$$
|p(z, x, y)| \leq\|p(z / 2, x, .)\|_{2}\|p(z / 2, . ., y)\|_{2}
$$

and from (13).

4) Using (14) the proof of Theorem 2 follows by similar arguments as in the proofs of Theorems 7.2 and 7.3 in 24. The latter used Lemma 6.18 in 24, which now needs a small modification: instead of $F\left(r e^{i \theta}\right) \leq a(r \cos \theta)^{-\beta}$ in the assumptions, we now have $F\left(r e^{i \theta}\right) \leq a(r \cos (w+|\theta|))^{-\beta}$. The proof given there for that lemma works under the last assumption and leads to the same conclusion with $\cos \theta$ now replaced by $\cos (w+|\theta|)$.

Corollary 3. For every $p \in[1, \infty]$ and every $\varepsilon>0$ there exists a positive constant $C_{\varepsilon}$ such that

$$
\left\|e^{-z A}\right\|_{\mathcal{L}\left(L^{1}\right)} \leq \frac{C_{\varepsilon}}{[\cos (w+|\theta|)]^{d\left|\frac{1}{2}-\frac{1}{p}\right|+\varepsilon}}
$$

for all $z=|z| e^{i \theta} \in \Sigma(\pi / 2-w)$. Here $d$ is as in (2).

Proof. It is enough to treat the case $p=1$. The general case follows from (10) and the Riesz-Thorin interpolation theorem.

The proof is very similar to that of Theorem 7.4 in [24]. From Theorem 2, we have

$$
\begin{aligned}
|p(z, x, y)| & \leq \frac{C^{\prime}}{\left[v\left(x, t_{0}^{1 / m}\right) v\left(y, t_{0}^{1 / m}\right)\right]^{1 / 2}} \\
& \times \exp \left[-c^{\prime} \frac{\rho(x, y)^{m /(m-1)}}{t_{0}^{1 /(m-1)}} \cos (w+|\theta|)^{m /(m-1)}\right],
\end{aligned}
$$

where $t_{0}=|z| \cos (w+|\theta|)$. Using this estimate and (12) we obtain

$$
\int_{\Omega}|p(z, x, y)| d \mu(y) \leq \frac{C_{1}}{[\cos (w+|\theta|)]^{d}} .
$$

Fix $\varepsilon \in(0,1)$ and let $p_{\varepsilon}$ be such that $1=\frac{1-\varepsilon}{2}+\frac{\varepsilon}{p_{\varepsilon}}$. By Hölder's inequality

$$
\int_{\Omega}|p(z, x, y)| d \mu(y) \leq\left(\int_{\Omega}|p(z, x, y)|^{2} d \mu(y)\right)^{\frac{1-\varepsilon}{2}}\left(\int_{\Omega}|p(z, x, y)|^{p_{\varepsilon}} d \mu(y)\right)^{\frac{1+\varepsilon}{2}} .
$$

We use (13) to estimate the first term on the RHS and use Theorem 2 to estimate the second one. We put this together and obtain

$$
\int_{\Omega}|p(z, x, y)| d \mu(y) \leq \frac{C_{\varepsilon}}{[\cos (w+|\theta|)]^{d / 2+\varepsilon d / 2}} .
$$

(See the proof of Theorem 7.4 in 24] for more details.) This gives

$$
\left\|e^{-z A}\right\|_{\mathcal{L}\left(L^{\infty}\right)} \leq \frac{C_{\varepsilon}}{[\cos (w+|\theta|)]^{d / 2+\varepsilon d / 2}} .
$$


The same estimate applies to the adjoint semigroup $e^{-z A^{*}}$, and this gives by duality the $L^{1}$-estimate.

As we already mentioned, the semigroup $\left(e^{-t A}\right)_{t \geq 0}$ extends to $L^{p}(\Omega, \mu)$ for $1 \leq$ $p<\infty$ and is bounded holomorphic with the same sector of holomorphy as on $L^{2}(\Omega, \mu)$ (i.e., $\left.\Sigma(\pi / 2-w)\right)$. This follows from Corollary 3 together with Vitali's theorem as in the symmetric case (see [24], Corollary 7.5).

\section{RiESZ MEANS AND BOUNDARY VALUES OF THE SEMIGROUP ON $L^{p}$}

We have seen in (11) and the comments following it that the boundary value of the semigroup $e^{-z A}$ acts on $L^{2}(\Omega, \mu)$. More precisely, $\left(e^{-t e^{i(\pi / 2-w)} A}\right)_{t \geq 0}$ and $\left(e^{-t e^{-i(\pi / 2-w)} A}\right)_{t \geq 0}$ are strongly continuous contraction semigroups on $L^{2}(\Omega, \mu)$. Their generators are $-e^{i(\pi / 2-w)} A$ and $-e^{-i(\pi / 2-w)} A$, respectively. If $A$ is selfadjoint (i.e., $w=0$ ), the above two semigroups are in fact the two branches of the strongly continuous Schrödinger group $\left(e^{-i t A}\right)_{t \in \mathbb{R}}$. A natural question is whether $\left(e^{-t e^{ \pm i(\pi / 2-w)} A}\right)_{t \geq 0}$ also act on $L^{p}(\Omega, \mu)$ for $p \neq 2$. In the case of the Laplace operator $A=-\Delta$ on $L^{2}\left(\mathbb{R}^{d}, d x\right)$, a well known result of Hörmander says that the Schrödinger group $e^{i t \Delta}$ acts on $L^{p}\left(\mathbb{R}^{d}, d x\right)$ only if $p=2$. The $L^{p}$-properties of $e^{i t \Delta}$ have been studied by several authors. Sjöstrand [25] proved that the Riesz-mean $I_{\alpha}(t):=t^{-\alpha} \int_{0}^{t}(t-s)^{\alpha-1} e^{-i s \Delta} d s$ acts as a bounded operator on $L^{p}\left(\mathbb{R}^{d}, d x\right)$ (with the $L^{p}$-norm bounded by a constant independent of $\left.t\right)$, provided $\alpha>d\left|\frac{1}{2}-\frac{1}{p}\right|$ and $1 \leq p<\infty$. This result was extended by many authors to elliptic operators; see [2], 14], 18]. The most general result, saying that this holds for all self-adjoint operators for which (4) holds, was proved in [5]. See also [24], Chapter 7. Another interesting result concerning the Schrödinger group is that $(I-\Delta)^{-\alpha} e^{-i t \Delta} \in$ $\mathcal{L}\left(L^{p}\left(\mathbb{R}^{d}, d x\right)\right)$, again provided that $\alpha>d\left|\frac{1}{2}-\frac{1}{p}\right|$. This is due to Lanconelli [22]. Similar results of this type have been obtained in [14, [4], 5] and [24] for all selfadjoint operators that satisfy (4). In this section we show non-symmetric versions of these results. More precisely, we prove the following.

Theorem 4. Assume that (2), (3) and (41) are satisfied. Let $\psi:=\pi / 2-w$ and $p \in[1, \infty)$.

1) Define the Riesz mean operator

$$
I_{\alpha}(t):=t^{-\alpha} \int_{0}^{t}(t-s)^{\alpha-1} e^{-s e^{i \psi} A} d s, \quad t>0 .
$$

Then, for every $\alpha>d\left|\frac{1}{2}-\frac{1}{p}\right|, I_{\alpha}(t) \in \mathcal{L}\left(L^{p}(\Omega)\right)$ with $\left\|I_{\alpha}(t)\right\|_{\mathcal{L}\left(L^{p}\right)} \leq C$ for all $t>0$, where $C$ is a positive constant.

The same conclusion holds for $I_{\alpha}^{\prime}(t):=t^{-\alpha} \int_{0}^{t}(t-s)^{\alpha-1} e^{-s e^{-i \psi} A} d s$.

2) For every $\alpha>d\left|\frac{1}{2}-\frac{1}{p}\right|$, the operators $(I+A)^{-\alpha} e^{-t e^{i \psi} A}$ and $(I+A)^{-\alpha} e^{-t e^{-i \psi} A}$ are bounded on $L^{p}(\Omega, \mu)$ with $L^{p}$ - norms bounded by $C(1+t)^{\alpha}(C$ is again a positive constant).

Proof. 1) The proof of this assertion is similar to the case of self-adjoint operators [14, 4], 5] and 24]. We shall however give the full details of the proof.

Define $J_{\alpha}(z):=\int_{[0, z]}(z-\xi)^{\alpha-1} e^{-\xi A} d \xi$ for $z \in \Sigma(\psi)$. We prove that for $\alpha>$ $d\left|\frac{1}{2}-\frac{1}{p}\right|$

$$
\left\|J_{\alpha}(z)\right\|_{\mathcal{L}\left(L^{p}\right)} \leq C|z|^{\alpha} .
$$


Once this is proved, we obtain the first assertion from strong continuity of $e^{-\xi A}$ on $L^{2}$ and the fact that $J_{\alpha}\left(t e^{i \psi}\right)=e^{\alpha i \psi} t^{\alpha} I_{\alpha}(t)$.

As mentioned in the previous section, $\xi \rightarrow e^{-\xi A}$ is holomorphic on $\Sigma(\psi)$, and hence $J_{\alpha}(z)$ does not depend on the path of integration (from 0 to $z$ ). Thus we can write $J_{\alpha}(z)=J_{\alpha}^{1}(z)+J_{\alpha}^{2}(z)$, where the first term consists of integrating on $[0,|z|]$ and the second on the part of the circle of center 0 and radius $|z|$.

Using the fact that $\left\|e^{-s A}\right\|_{\mathcal{L}\left(L^{p}\right)} \leq C$ (which follows from (11) and (4) as mentioned in the previous section), we have

$$
\begin{aligned}
\left\|J_{\alpha}^{1}(z)\right\|_{\mathcal{L}\left(L^{p}\right)} & =\left\|\int_{0}^{|z|}(z-s)^{\alpha-1} e^{-s A} d s\right\|_{\mathcal{L}\left(L^{p}\right)} \\
& \leq C \int_{0}^{|z|}|z-s|^{\alpha-1} d s \\
& \leq C^{\prime}|z|^{\alpha} .
\end{aligned}
$$

Concerning $J_{\alpha}^{2}(z)$, we use Corollary 3 and we have (here $\theta=\arg z$ )

$$
\begin{aligned}
\left\|J_{\alpha}^{2}(z)\right\|_{\mathcal{L}\left(L^{p}\right)} & =\left\|\int_{0}^{\theta}\left(z-|z| e^{i \varphi}\right)^{\alpha-1} e^{-|z| e^{i \varphi} A}|z| i e^{i \varphi} d \varphi\right\|_{\mathcal{L}\left(L^{p}\right)} \\
& \leq C_{\varepsilon}|z| \int_{0}^{\theta}|z-| z\left|e^{i \varphi}\right|^{\alpha-1} \cos (w+\varphi)^{-d\left|\frac{1}{2}-\frac{1}{p}\right|-\varepsilon} d \varphi \\
& \leq C_{\varepsilon}|z|^{\alpha} \int_{0}^{\theta} \sin \left(\frac{\theta-\varphi}{2}\right)^{\alpha-1} \cos (w+\varphi)^{-d\left|\frac{1}{2}-\frac{1}{p}\right|-\varepsilon} d \varphi .
\end{aligned}
$$

Since $\sin \left(\frac{\theta-\varphi}{2}\right) \leq \sin \left(\frac{\pi / 2-w-\varphi}{2}\right) \leq \cos (w+\varphi)$ we obtain that for $\alpha>d\left|\frac{1}{2}-\frac{1}{p}\right|$,

$$
\begin{aligned}
\left\|J_{\alpha}^{2}(z)\right\|_{\mathcal{L}\left(L^{p}\right)} & \leq C_{\varepsilon}|z|^{\alpha} \int_{0}^{\theta} \sin \left(\frac{\theta-\varphi}{2}\right)^{\alpha-1-d\left|\frac{1}{2}-\frac{1}{p}\right|-\varepsilon} d \varphi \\
& \leq C_{\varepsilon}|z|^{\alpha} \int_{0}^{\pi / 4} \sin (\nu)^{\alpha-1-d\left|\frac{1}{2}-\frac{1}{p}\right|-\varepsilon} d \nu \\
& \leq C_{\varepsilon}^{\prime}|z|^{\alpha} .
\end{aligned}
$$

This proves (16).

2) Fix $t>0$. First, note that

$$
\Gamma(\alpha)(I+A)^{-\alpha} e^{-t e^{i \psi} A}=\int_{0}^{\infty} e^{-s} s^{\alpha-1} e^{-\left(s+t e^{i \psi}\right) A} d s .
$$

The RHS can also be rewritten as

$$
\int_{\gamma_{t}} e^{-z+t e^{i \psi}}\left(z-t e^{i \psi}\right)^{\alpha-1} e^{-z A} d z
$$

where $\gamma_{t}$ is the line $\left\{z=s+t e^{i \psi}, s \geq 0\right\}$. By analyticity and the Cauchy formula, the integral over $\gamma_{t}$ coincides with the sum of the integrals over $(+\infty, t)$ and $\gamma_{\psi}$, where $\gamma_{\psi}$ is the part of the circle of center 0 and radius $t$. The first term is given by

$$
T_{1}:=\int_{t}^{\infty} e^{-s+t e^{i \psi}}\left(s-t e^{i \psi}\right)^{\alpha-1} e^{-s A} d s .
$$

Using the fact that $\left\|e^{-s A}\right\|_{\mathcal{L}\left(L^{p}\right)} \leq C$, we easily obtain that

$$
\left\|T_{1}\right\|_{\mathcal{L}\left(L^{p}\right)} \leq C^{\prime}(1+t)^{\alpha} e^{-(1-\sin w) t} \leq C^{\prime \prime} .
$$


The second term is

$$
T_{2}:=\int_{0}^{\psi} e^{t e^{i \theta}-t e^{i \psi}}\left(t e^{i \theta}-t e^{i \psi}\right)^{\alpha-1} e^{-t e^{i \theta} A} t e^{i \theta} i d \theta .
$$

Therefore, again using Corollary 3 we obtain for $\beta>d\left|\frac{1}{2}-\frac{1}{p}\right|$,

$$
\begin{aligned}
\left\|T_{2}\right\|_{\mathcal{L}\left(L^{p}\right)} & \leq C t^{\alpha} \int_{0}^{\psi} \frac{\left|1-e^{i(\psi-\theta)}\right|^{\alpha-1}}{[\cos (w+\theta)]^{\beta}} d \theta \\
& =C t^{\alpha} \int_{0}^{\pi / 2-w} \frac{[\sin (\pi / 4-w / 2-\theta / 2)]^{\alpha-1}}{[\cos (w+\theta)]^{\beta}} d \theta \\
& =C t^{\alpha} \int_{0}^{\pi / 2-w} \frac{[\sin (\theta / 2)]^{\alpha-1}}{[\sin (\theta)]^{\beta}} d \theta .
\end{aligned}
$$

The last term is bounded by $C^{\prime} t^{\alpha}$ for $\alpha>\beta$. This proves that $(I+A)^{-\alpha} e^{-t e^{i \psi} A}$ is bounded on $L^{p}$ and

$$
\left\|(I+A)^{-\alpha} e^{-t e^{i \psi} A}\right\|_{\mathcal{L}\left(L^{p}\right)} \leq C\left(1+t^{\alpha}\right)
$$

for all $\alpha>d\left|\frac{1}{2}-\frac{1}{p}\right|$. The same proof shows that this also holds for $(I+A)^{-\alpha} e^{-t e^{-i \psi} A}$.

The last result can be reformulated in terms of integrated semigroups and allows one to treat the Cauchy problem

$$
\begin{cases}\frac{d u}{d t} & =-e^{i(\pi / 2-w)} A u(t), t \geq 0 \\ u(0) & =f\end{cases}
$$

in $L^{p}(\Omega, \mu)$ for initial data $f \in D\left(A^{\beta}\right)$ for appropriate $\beta$. See [3], [14, [18, [5].

\section{The MUltiPlieR THEOREM}

Let $A: D(A) \subseteq L^{2}(\Omega, \mu) \rightarrow L^{2}(\Omega, \mu)$ as in the previous sections. We need some other notation. Following [7, Theorem 4.10, we define for $x \in \mathbb{R}$

$$
\begin{aligned}
\phi_{0}(x) & :=(2-2|x|)_{+}-(1-2|x|)_{+}, \\
\phi_{1}(x) & :=(1-2|x-1|)_{+}+(1 / 2-|x-3 / 2|)_{+},
\end{aligned}
$$

and

$$
\phi_{\varepsilon n}(x):=\phi_{1}\left(2^{1-n} \varepsilon x\right) \text { for all positive integers } n \text { and } \varepsilon \in\{-1,1\} .
$$

Let $f \in H^{\infty}(\Sigma(w))$ and continuous on $\overline{\Sigma(w)}$. It is shown in [9] (see also [8] for a more general result) that $f(A)$ is well defined and bounded on $L^{2}(\Omega, \mu)$ with

$$
\|f(A)\|_{\mathcal{L}\left(L^{2}(\Omega)\right)} \leq c\|f\|_{w},
$$

where $c$ is a universal constant ( $c$ can even be chosen to be $\left.2+\frac{2}{\sqrt{3}}\right)$. We now wish to extend $f(A)$ to $L^{p}$ for $p \neq 2$ (under a condition on the behavior of $f$ on the boundary of the sector $\Sigma(w))$.

We denote by $f_{+w, e}$ and $f_{-w, e}$ the functions defined by

$$
f_{ \pm w, e}(\lambda):=f\left(e^{\lambda} e^{ \pm i w}\right) .
$$


The two functions $f_{ \pm w, e}$ are continuous on $\mathbb{R}$. We define the convolution $f_{ \pm w, e} * \check{\phi_{n}}$ (here $\check{\phi_{n}}$ denotes the inverse Fourier transform). We have

Theorem 5. Assume that (11), (3) and (4) hold. Let $f \in H^{\infty}(\Sigma(w))$ and continuous on $\overline{\Sigma(w)}$. If $f$ satisfies

$$
\sum_{n \in \mathbb{Z}} 2^{|n| \alpha} \sup _{x \in \mathbb{R}}\left|f_{ \pm w, e} * \check{\phi_{n}}(x)\right|<\infty
$$

for some constant $\alpha>\frac{d}{2}+1$, then $f(A)$ is of weak type $(1,1)$. In particular $f(A)$ is bounded on $L^{p}(\Omega, \mu)$ for all $p \in(1, \infty)$.

If (19) holds for smaller $\alpha$, then one obtains that $f(A)$ is bounded on $L^{p}(\Omega, \mu)$ for $p$ in a certain interval around 2. More precisely,

Theorem 6. Assume that (11), (3) and (4) hold. Fix $p \in(1,2)$. Let $f \in H^{\infty}(\Sigma(w))$ and continuous on $\overline{\Sigma(w)}$. If $f$ satisfies

$$
\sum_{n \in \mathbb{Z}} 2^{|n| \alpha_{p}} \sup _{x \in \mathbb{R}}\left|f_{ \pm w, e} * \check{\phi_{n}}(x)\right|<\infty,
$$

for some constant $\alpha_{p}>\left[\frac{d}{2}+1\right]|1-2 / p|$, then $f(A)$ is bounded on $L^{r}(\Omega, \mu)$ for all $r \in\left[p, \frac{p}{p-1}\right]$.

The proofs of these results rely heavily on sharp estimates for the holomorphic functional calculus. This will be done in the next section.

Now we explain how Theorem 1 can be deduced from these results. All the arguments here are taken from [7. Denote by $D$ the derivation and by $I$ the integration on $\mathbb{R}\left(I g(x)=\int_{-\infty}^{x} g(t) d t\right)$; then for any $k \in \mathbb{N}$,

$$
f_{ \pm w, e} * \check{\phi_{n}}=D^{k} f_{ \pm w, e} * I^{k} \check{\phi_{n}} .
$$

One also obtains from the definition of $\phi_{n}$

$$
\left\|I^{k} \check{\phi}_{n}\right\|_{1} \leq C_{k} 2^{-k|n|}
$$

Thus, if $f$ is as in Theorem 1, we choose $k=\left[\frac{d}{2}+1\right]|1-2 / p|+1$ and note that all the derivatives up to $k$ of $f_{ \pm w, e}$ are bounded. Now we use the fact that $f_{ \pm w, e}=$ $\sum_{n \in \mathbb{Z}} f_{ \pm w, e} * \check{\phi_{n}}$ and obtain (20) (or (19) if $p=1$ ), from which we conclude that $f(A)$ is bounded on $L^{p}(\Omega, \mu)$ if $p \in(1,2)$ (and of weak type $(1,1)$ if $p=1$ ). Since $f(A)$ is bounded on $L^{2}(\Omega, \mu)$ one has by interpolation and duality that $f(A) \in \mathcal{L}\left(L^{r}(\Omega)\right)$ for $r \in\left[p, \frac{p}{p-1}\right]$ if $p \in(1, \infty)$ (and $f(A) \in \mathcal{L}\left(L^{r}(\Omega)\right.$ ) for all $r \in(1, \infty)$ if $p=1$ ).

When $w=0$, the space $\Lambda_{\infty, 1}^{\alpha}$ of functions satisfying (19) is a Besov space. The condition on $f$ in the previous theorems is that $f$ is bounded holomorphic inside $\Sigma(w)$ and belongs to a Besov space on the boundary. Some interesting information and reformulations of function in $\Lambda_{\infty, 1}^{\alpha}$ can be found in Galé and Miana [15].

\section{The Proof of the MUltiplier theorem}

In order to prove Theorem [5] we shall use the holomorphic functional calculus introduced by McIntosh [23. As mentioned in the Introduction, it follows from [1] and [12] that under the conditions (11), (4) and (3) the operator $A$ has a bounded holomorphic functional calculus on $L^{p}(\Omega, \mu)$ for every $p \in(1, \infty)$. Here we need 
sharp estimates for $\|f(A)\|_{\mathcal{L}\left(L^{p}\right)}$ in terms of $\nu$ for $f \in H^{\infty}(\Sigma(w+\nu))$. We prove the following

Theorem 7. Assume that (11), (3) and (4) hold. Fix $\nu>0$ such that $w+\nu<\pi / 2$. For every $f \in H^{\infty}(\Sigma(w+\nu))$, the operator $f(A)$ is of weak type $(1,1)$ with the weak type $(1,1)$ estimate

$$
\|f(A)\|_{L^{1}, L^{1, w}} \leq \frac{C}{\nu^{\alpha}}\|f\|_{w+\nu}
$$

for every $\alpha>\frac{d}{2}+1$, where $C$ is a positive constant independent of $f$ and $\nu$,

Proof. We fix $\nu>0$ as above, $\alpha>\frac{d}{2}+1$, and $f \in H^{\infty}(\Sigma(w+\nu))$.

As in the proof of Theorem 6 in [12], we may assume that $f$ decays at 0 and infinity and conclude by an approximation argument. In this case, $f(A)$ is defined by

$$
f(A):=\frac{1}{2 \pi i} \int_{\gamma} f(\lambda)(A-\lambda I)^{-1} d \lambda,
$$

where $\gamma$ is a clockwise parametrized contour $\left\{\lambda=t e^{ \pm i(w+\nu)}, t \geq 0\right\}$. We work with $f(A)$ as a bounded operator on $L^{2}(\Omega, \mu)$ and prove that it is of weak type $(1,1)$ with the desired weak type $(1,1)$ estimate.

Again as in [12, by using the fact that the resolvent is the Laplace transform of the semigroup, we have

$$
f(A)=\int_{\Gamma_{+}} e^{-z A} g_{+}(z) d z+\int_{\Gamma_{-}} e^{-z A} g_{-}(z) d z,
$$

where $g_{ \pm}$are given by

$$
g_{ \pm}(z):=\int_{\gamma_{ \pm}} f(\lambda) e^{\lambda z} d \lambda
$$

Here $\Gamma_{ \pm}$and $\gamma_{ \pm}$are the contours $\Gamma_{ \pm}:=\left\{s e^{ \pm i(\pi / 2-w-\nu / 2)}, s \geq 0\right\}$ and $\gamma_{ \pm}:=$ $\left\{s e^{ \pm i(w+\nu)}, s \geq 0\right\}$. The operator $f(A)$ is a singular integral operator. Its associated kernel is given by

$$
k_{f}(x, y)=\int_{\Gamma_{+}} p(z, x, y) g_{+}(z) d z+\int_{\Gamma_{-}} p(z, x, y) g_{-}(z) d z:=\int_{\Gamma_{ \pm}} p(z, x, y) g_{ \pm}(z) d z .
$$

Let $f_{u}(\lambda):=e^{-\lambda u} f(\lambda)$. We prove that for some constant $C$ independent of $f$ and $\nu$,

$$
\int_{\rho(x, y) \geq u^{1 / m}}\left|k_{f}(x, y)-k_{f_{u}}(x, y)\right| d \mu(x) \leq C \nu^{-\alpha}\|f\|_{w+\nu}, u>0 \text { (a.e.) } y \in \Omega .
$$

Once this is proved, we obtain from Theorems 1 and 2 in [12] (see also Theorems 7.21 and 7.22 in $[24])$ that $f(A)$ is of weak type $(1,1)$ with the weak type $(1,1)$ estimate

$$
\|f(A)\|_{L^{1}, L^{1, w}} \leq C^{\prime}\left[\nu^{-\alpha}\|f\|_{w+\nu}+\|f(A)\|_{\mathcal{L}\left(L^{2}(\Omega)\right)}\right] .
$$

As mentioned in (17), by a result in [9] or [8] we have $\|f(A)\|_{\mathcal{L}\left(L^{2}(\Omega)\right)} \leq c\|f\|_{w+\nu}$ with a universal constant $c$. One then obtains the desired weak type $(1,1)$ estimate of Theorem 7. It then remains to prove (24). For this reason we shall use (23). The 
two terms there are similar, and hence we only handle one of them. If we consider for example the first one, then we have to prove that

$$
\int_{\rho(x, y) \geq u^{1 / m}}\left|\int_{\Gamma_{+}} p(z, x, y)\left[g_{+}(z)-\left(g_{u}\right)_{+}(z)\right] d z\right| d \mu(x) \leq C \nu^{-\alpha}\|f\|_{w+\nu},
$$

where $\left(g_{u}\right)_{+}:=\int_{\gamma_{+}} f_{t}(\lambda) e^{\lambda z} d \lambda$. Set $z=t e^{i(\pi / 2-w-\nu / 2)}$. We have

$$
\begin{aligned}
g_{+}(z)-\left(g_{u}\right)_{+}(z) & =\int_{\gamma_{+}} f(\lambda)\left[1-e^{-u \lambda}\right] e^{\lambda z} d \lambda \\
& =\int_{0}^{\infty} f\left(s e^{i(w+\nu)}\right)\left[1-e^{-u s e^{i(w+\nu)}}\right] e^{s t e^{i(\pi / 2+\nu / 2)}} d s e^{i(w+\nu)} .
\end{aligned}
$$

Since $w+\nu<\pi / 2$, it is easy to see that

$$
\left|1-e^{-u s e^{i(w+\nu)}}\right| \leq 2 \inf (1, u s) .
$$

Then,

$$
\left|g_{+}(z)-\left(g_{u}\right)_{+}(z)\right| \leq 2\|f\|_{w+\nu} \int_{0}^{\infty} \inf (1, u s) e^{-s t \sin (\nu / 2)} d s .
$$

Therefore,

$$
\left|g_{+}(z)-\left(g_{u}\right)_{+}(z)\right| \leq \frac{2}{u}\|f\|_{w+\nu}\left[\int_{0}^{1} s e^{-\frac{s t}{u} \sin (\nu / 2)} d s+\int_{1}^{\infty} e^{-\frac{s t}{u} \sin (\nu / 2)} d s\right] .
$$

By Theorem 2, we have for $\rho(x, u) \geq u^{1 / m}$ and $z=t e^{i(\pi / 2-w-\nu / 2)}$

$$
\begin{aligned}
& |p(z, x, y)| \leq \frac{C^{\prime}}{\left[v\left(x,\left(\frac{t \sin (\nu / 2)}{\cos w}\right)^{1 / m}\right) v\left(y,\left(\frac{t \sin (\nu / 2)}{\cos w}\right)^{1 / m}\right)\right]^{1 / 2}} \\
& \quad \times \exp \left[-\frac{c^{\prime}}{2} \frac{\rho(x, y)^{m /(m-1)}}{t^{1 /(m-1)}} \sin (\nu / 2)\right] \exp \left[-\frac{c^{\prime}}{2}\left(\frac{u}{t}\right)^{1 /(m-1)} \sin (\nu / 2)\right] .
\end{aligned}
$$

Integrating this we have as for (15)

$$
\int_{\rho(x, y) \geq u^{1 / m}}|p(z, x, y)| d \mu(x) \leq \frac{C_{1}}{(\sin (\nu / 2))^{d}} \exp \left[-\frac{c^{\prime}}{2}\left(\frac{u}{t}\right)^{1 /(m-1)} \sin (\nu / 2)\right] .
$$

Using this estimate, Corollary 3 and the trivial inequality

$$
\begin{aligned}
\int_{\rho(x, y) \geq u^{1 / m}}|p(z, x, y)| d \mu(x) & \leq\left(\int_{\rho(x, y) \geq u^{1 / m}}|p(z, x, y)| d \mu(x)\right)^{\delta} \\
& \times\left(\int_{\Omega}|p(z, x, y)| d \mu(x)\right)^{1-\delta} \quad(\forall \delta>0),
\end{aligned}
$$

we obtain that for every $\varepsilon>0$

(28)

$$
\int_{\rho(x, y) \geq u^{1 / m}}|p(z, x, y)| d \mu(x) \leq \frac{C_{\varepsilon}^{\prime}}{(\sin (\nu / 2))^{d / 2+\varepsilon}} \exp \left[-c_{\varepsilon}\left(\frac{u}{t}\right)^{1 /(m-1)} \sin (\nu / 2)\right] .
$$

From this and (27) we obtain the estimate

$$
\int_{\rho(x, y) \geq u^{1 / m}}\left|\int_{\Gamma_{+}} p(z, x, y)\left[g_{+}(z)-\left(g_{u}\right)_{+}(z)\right] d z\right| d \mu(x) \leq \frac{C_{\varepsilon}^{\prime \prime}\|f\|_{w+\nu}}{(\sin (\nu / 2))^{d / 2+\varepsilon}}\left[I_{1}+I_{2}\right],
$$


where

$$
I_{1}:=\frac{1}{u} \int_{0}^{\infty} \int_{0}^{1} s e^{-\frac{s t}{u} \sin (\nu / 2)} \exp \left[-c_{\varepsilon}\left(\frac{u}{t}\right)^{1 /(m-1)} \sin (\nu / 2)\right] d s d t
$$

and

$$
I_{2}:=\frac{1}{u} \int_{0}^{\infty} \int_{1}^{\infty} e^{-\frac{s t}{u} \sin (\nu / 2)} \exp \left[-c_{\varepsilon}\left(\frac{u}{t}\right)^{1 /(m-1)} \sin (\nu / 2)\right] d s d t .
$$

Consider $I_{1}$. We make the change of variable $\xi=\frac{t}{u}$ and obtain

$$
\begin{aligned}
I_{1} & =\int_{0}^{\infty} \int_{0}^{1} s e^{-s \xi \sin (\nu / 2)} \exp \left[-c_{\varepsilon} \xi^{-1 /(m-1)} \sin (\nu / 2)\right] d s d \xi \\
& \leq 1+\int_{1}^{\infty} \int_{0}^{1} s e^{-s \xi \sin (\nu / 2)} \exp \left[-c_{\varepsilon} \xi^{-1 /(m-1)} \sin (\nu / 2)\right] d s d \xi \\
& \leq 1+\int_{0}^{1} s \int_{1}^{\infty} e^{-s \xi \sin (\nu / 2)} d \xi d s \\
& \leq 1+\frac{1}{\sin (\nu / 2)} \int_{0}^{1} e^{-s \sin (\nu / 2)} d s \\
& \leq \frac{2}{\sin (\nu / 2)}
\end{aligned}
$$

The same estimate holds also for $I_{2}$. Indeed,

$$
\begin{aligned}
I_{2} & =\int_{0}^{\infty} \int_{1}^{\infty} e^{-s \xi \sin (\nu / 2)} \exp \left[-c_{\varepsilon} \xi^{-1 /(m-1)} \sin (\nu / 2)\right] d s d \xi \\
& =\frac{1}{\sin (\nu / 2)} \int_{0}^{\infty} \exp \left[-c_{\varepsilon} \xi^{-1 /(m-1)} \sin (\nu / 2)\right] e^{-\xi \sin (\nu / 2)} \frac{d \xi}{\xi} \\
& \leq \frac{1}{\sin (\nu / 2)}\left(\int_{0}^{1} \exp \left[-c_{\varepsilon} \xi^{-1 /(m-1)} \sin (\nu / 2)\right] \frac{d \xi}{\xi}+\int_{1}^{\infty} e^{-\xi \sin (\nu / 2)} \frac{d \xi}{\xi}\right) \\
& \leq \frac{C_{\delta}}{(\sin (\nu / 2))^{1+\delta}}(\forall \delta>0) .
\end{aligned}
$$

The last estimate is obtained from the obvious inequality $e^{-x} \leq C_{\delta} x^{-\delta}$ for all $x>0$ and $\delta>0$. Inserting these estimates in (29) we obtain (25). We have proved (24).

If we combine the previous theorem with the result mentioned in (17), we obtain by classical interpolation theorems the following corollary.

Corollary 8. For every fixed $\nu>0$ such that $w+\nu<\pi / 2$ and $p \in(1, \infty)$, the operator $f(A)$ is bounded on $L^{p}(\Omega, \mu)$ for every $f \in H^{\infty}(\Sigma(w+\nu))$ with norm estimate

$$
\|f(A)\|_{\mathcal{L}\left(L^{p}\right)} \leq \frac{C}{\nu^{\alpha_{p}}}\|f\|_{w+\nu}
$$

for every $\alpha_{p}>\left[\frac{d}{2}+1\right]|1-2 / p|$, where $C$ is a positive constant independent of $f$ and $\nu$.

A direct consequence of the above results is the following estimate for imaginary powers $A^{i s}$. 
Corollary 9. For every $s \in \mathbb{R}$, the operator $A^{\text {is }}$ is of weak type $(1,1)$ with

$$
\left\|A^{i s}\right\|_{L^{1}, L^{1, w}} \leq C_{\varepsilon} e^{w|s|}(1+|s|)^{d / 2+1+\varepsilon} \quad \forall s \in \mathbb{R} .
$$

Fix $p \in(1, \infty)$ and let $\alpha_{p}>\left[\frac{d}{2}+1\right]|1-2 / p|$. The operator $A^{i s}$ is bounded on $L^{p}(\Omega, \mu)$ with

$$
\left\|A^{i s}\right\|_{\mathcal{L}\left(L^{p}\right)} \leq C e^{w|s|}(1+|s|)^{\alpha_{p}} \forall s \in \mathbb{R} .
$$

Proof. We apply Theorem 7 and Corollary 8 with the function $f(z)=z^{i s}$ and obtain for every fixed $\alpha>\frac{d}{2}+1$ and $s \in \mathbb{R}$

$$
\left\|A^{i s}\right\|_{L^{1}, L^{1, w}} \leq \frac{C}{\nu^{\alpha}} e^{(w+\nu)|s|} \forall \nu>0,
$$

and for $p \in(1, \infty)$ and $\alpha_{p}>\left[\frac{d}{2}+1\right]|1-2 / p|$

$$
\left\|A^{i s}\right\|_{\mathcal{L}\left(L^{p}\right)} \leq \frac{C}{\nu^{\alpha_{p}}} e^{(w+\nu)|s|} \quad \forall \nu>0 .
$$

Therefore, Corollary 9 follows when $|s|$ is not large, say when $|s| \leq 1$. If $|s|>1$, we choose in these last two estimates $\nu=\frac{1}{|s|}$ and obtain the result.

The above corollary can also be obtained as a particular case of Theorems 5 and 6 , Now we show that these two theorems also follow from Theorem 7 and Corollary 8 . The idea that a spectral multiplier theorem follows from the holomorphic functional calculus is taken from [7, Theorem 4.10. It is assumed there that the spectrum of $A, \sigma(A)$ is contained in $[0, \infty)$. This corresponds in our situation to the self-adjoint case. We extend some arguments of [7] in order to treat non-self-adjoint operators.

Proofs of Theorems 5 and 6. We recall the following facts from [7. Let $g$ be a continuous bounded function on the real line $\mathbb{R}$. By the Paley-Wiener theorem, $g * \check{\phi_{n}}$ extends to an entire function $\mathcal{E}\left(g * \check{\phi_{n}}\right)$, and one obtains by the maximum principle

$$
\sup _{x \in \mathbb{R},|y| \leq b}\left|\mathcal{E}\left(g * \check{\phi_{n}}\right)(x+i y)\right| \leq e^{b 2^{|n|+1}} \sup _{x \in \mathbb{R}}\left|g * \check{\phi_{n}}(x)\right| .
$$

Assume now that $g$ is bounded holomorphic in the band $B(w):=\{z=x+i y \in$ $\mathbb{C},|y|<w\}$, and continuous on $\overline{B(w)}$. Denote again by $\mathcal{E}\left(g * \check{\phi_{n}}\right)$ its entire extension. By the Cauchy formula and analyticity of $g$ on $B(w)$ one has 2

$$
\begin{aligned}
\mathcal{E}\left(g * \check{\phi_{n}}\right)(x+i y) & =\int_{\mathbb{R}} g(t) \check{\phi_{n}}(x+i y-t) d t \\
& =\int_{\mathbb{R}+i w} g(\xi) \check{\phi_{n}}(x+i y-\xi) d \xi \\
& =\int_{\mathbb{R}} g_{w}(t) \check{\phi_{n}}(x+i(y-w)-t) d t \\
& =\left(g_{w} * \check{\phi_{n}}\right)(x+i(y-w)),
\end{aligned}
$$

\footnotetext{
${ }^{2}$ One may first apply the Cauchy formula for $g_{\varepsilon, \eta}(z):=e^{-\varepsilon z^{2}} g(\eta z)$ with $\eta, \varepsilon \in(0,1)$ and then let $\eta \rightarrow 1$ and $\varepsilon \rightarrow 0$.
} 
where $g_{w}(t):=g(t+i w)$. We obtain from (30) that

$$
\begin{aligned}
\sup _{x \in \mathbb{R},|y-w| \leq b}\left|\mathcal{E}\left(g * \check{\phi_{n}}\right)(x+i y)\right| & =\sup _{x \in \mathbb{R},|y-w| \leq b}\left|g_{w} * \check{\phi_{n}}(x+i(y-w))\right| \\
& \leq e^{b 2^{|n|+1}} \sup _{x \in \mathbb{R}}\left|g_{w} * \check{\phi_{n}}(x)\right| .
\end{aligned}
$$

In these arguments, we may change $w$ into $-w$, and hence

$$
\sup _{x \in \mathbb{R},|y \pm w| \leq b}\left|\mathcal{E}\left(g * \check{\phi_{n}}\right)(x+i y)\right| \leq e^{b 2^{|n|+1}} \sup _{x \in \mathbb{R}}\left|g_{ \pm w} * \check{\phi_{n}}(x)\right| .
$$

In particular, for every fixed $n$ we choose $b=2^{-|n|}$, and we have by the maximum principle

$$
\sup _{x \in \mathbb{R},|y| \leq w+2^{-|n|}}\left|\mathcal{E}\left(g * \check{\phi_{n}}\right)(x+i y)\right| \leq e^{2} \sup _{x \in \mathbb{R}}\left|g_{ \pm w} * \check{\phi_{n}}(x)\right| .
$$

Now let $A$ and $f$ be as in Theorems 5 or 6. Let $T:=\log (A)$. By [16], $\sigma(T) \subset B(w)$, and we obtain from Theorem 7 and Corollary 8 that

$$
\|g(T)\|_{L^{1}, L^{1, w}} \leq \frac{C}{\nu^{\alpha}} \sup _{z \in B(w+\nu)}|g(z)|
$$

and

$$
\|g(T)\|_{\mathcal{L}\left(L^{p}\right)} \leq \frac{C}{\nu^{\alpha_{p}}} \sup _{z \in B(w+\nu)}|g(z)|,
$$

for every small $\nu>0$ and every bounded holomorphic function $g$ on $B(w+\nu)$. We write $f_{e}(\lambda):=f\left(e^{\lambda}\right)$ for $\lambda \in B(w)$. We obtain from these estimates and (32)

$$
\left\|f_{e} * \check{\phi_{n}}(T)\right\|_{L^{1}, L^{1, w}} \leq C e^{2} 2^{\alpha|n|} \sup _{x \in \mathbb{R}}\left|f_{ \pm w, e} * \check{\phi_{n}}(x)\right|,
$$

and similarly for $\left\|f_{e} * \check{\phi_{n}}(T)\right\|_{\mathcal{L}\left(L^{p}\right)}$ with $\alpha_{p}$ in place of $\alpha\left(f_{ \pm w, e}\right.$ was defined in (18) $)$. The proofs of Theorem 5 and 6 are finished by observing that

$$
f_{e}=\sum_{n \in \mathbb{Z}} f_{e} * \check{\phi_{n}},
$$

together with the obvious fact that $f(A)=f_{e}(T)$. Note that the fact that $F_{N}(A):=$ $\sum_{n=-N}^{N} f_{e} * \check{\phi_{n}}(A)$ converges strongly to $f(A)$ on $L^{2}(\Omega, \mu)$ follows from the boundedness with respect to $N$ of $\left\|F_{N}(A)\right\|_{\mathcal{L}\left(L^{2}\right)}$ and the convergence lemma in [7].

\section{REFERENCES}

[1] G. Alexopoulos, Spectral multipliers on Lie groups of polynomial growth, Proc. Amer. Math. Soc. 120 (1994), no. 3, 973-979. MR.1172944 (95j:22016)

[2] G. Alexopoulos and N. Lohoué, Riesz means on Lie groups and Riemannian manifolds of nonnegative curvature, Bull. Soc. Math. France 122 (1994), no. 2, 209-223. MR:1273901 (95h:43011)

[3] W. Arendt, J.K. Batty, M. Hieber and F. Neubrander, Vector-Valued Laplace Transforms and Cauchy Problems, Monographs in Mathematics, vol 96, Birkhäuser 2001. MR 1886588 (2003g:47072)

[4] K. Boyadzhiev and R. deLaubenfels, Boundary values of holomorphic semigroups, Proc. Amer. Math. Soc. 118 (1993), no. 1, 113-118. MR1128725 (93f:47043)

[5] G. Carron, Th. Coulhon and E.M. Ouhabaz, Gaussian estimates and $L^{p}$-boundedness of Riesz means, J. Evol. Equ. 2 (2002), no. 3, 299-317. MR1930609(2003i:35116)

[6] Th. Coulhon and X.T. Duong, Riesz transforms for $1 \leq p \leq 2$, Trans. Amer. Math. Soc. 351 (1999), no. 3, 1151-1169. MR1458299 (99e:58174) 
[7] M. Cowling, I. Doust, A. McIntosh and A. Yagi, Banach space operators with a bounded $H^{\infty}$ functional calculus, J. Austral. Math. Soc. Ser. A 60 (1996), no. 1, 51-89. MR.1364554 (97d:47023)

[8] M. Crouzeix and B. Delyon, Some estimates for analytic functions of strip or sectorial operators, Arch. Math. 81 (2003), no. 5, 559-566. MR2029717(2004i:47009)

[9] B. Delyon and F. Delyon, Generalization of von Neumann's spectral sets and integral representation of operators, Bull. Soc. Math. France 127 (1999), no. 1, 25-41. MR1700467 (2000e:47017)

[10] X.T. Duong, From the $L^{1}$ norms of the complex heat kernels to a Hörmander multiplier theorem for sub-Laplacians on nilpotent Lie groups, Pacific J. Math. 173 (1996), no. 2, 413424. MR.1394398 (97h:43002)

[11] X.T. Duong and D.W. Robinson, Semigroup kernels, Poisson bounds, and holomorphic functional calculus, J. Funct. Anal. 142 (1996), no. 1, 89-128. MR1419418 (97j:47056)

[12] X.T. Duong and A. McIntosh, Singular integral operators with non-smooth kernels on irregular domains, Rev. Mat. Iberoamericana 15 (1999), no. 2, 233-265. MR 1715407|(2001e:42017a)

[13] X.T. Duong, E.M. Ouhabaz and A. Sikora, Plancherel-type estimates and sharp spectral multipliers, J. Funct. Anal. 196 (2002), no. 2, 443-485. MR1943098 (2003k:43012)

[14] O. El Mennaoui, Trace des Semi-groupes Holomorphes Singuliers à l'Origine et Comportement Asymptotique, Ph.D. Thesis, Université de franche-Comté, Besançon 1992.

[15] J. E. Galé and P.J. Miana, $H^{\infty}$-functional calculus and Mikhlin-type multiplier conditions, preprint, Universidad de Zaragoza 2006.

[16] M. Haase, Spectral properties of operator logarithms, Math. Z. 245 (2003), no. 4, 761-779. MR2020710 (2005b:47034)

[17] W. Hebisch, Functional calculus for slowly decaying kernels, preprint 1995.

[18] M. Hieber, Integrated semigroups and differential operators on $L^{p}$ spaces, Math. Ann. 291 (1991), no. 1, 1-16. MR.1125004 (92g:47052)

[19] E. Hille and R.S. Phillips, Functional Analysis and Semigroups, Coll. Publ. 31, American Math. Society 1957. MR0089373 (19:664d)

[20] L. Hörmander, Estimates for translation invariant operators in $L^{p}$ spaces, Acta. Math. 104 (1960) 93-140. MR0121655 (22:12389)

[21] T. Kato, Perturbation Theory for Linear Operators, 2nd edition, Springer-Verlag 1980.

[22] E. Lanconelli, Valuationi in $L^{p}\left(\mathbb{R}^{n}\right)$ della soluzione del problema di Cauchy per l'equazione di Schrödinger, Bull. Un. Mat. Ital. 4 (1968) 591-607. MR0234133 (38:2452)

[23] A. McIntosh, Operators which have an $H^{\infty}$ functional calculus, Mini-conference on Operator Theory and Partial Differential Equations, Proc. of the CMA, Australian National University, 14 (1986) 210-231. MR912940 (88k:47019)

[24] E.M. Ouhabaz, Analysis of Heat Equations on Domains, London Math. Soc Monographs 31, Princeton Univ. Press (2004). MR2124040 (2005m:35001)

[25] J. Sjöstrand, On the Riesz means of the solutions of the Schrödinger equation, Ann. Scuola Norm. Sup. Pisa 24 (1970) 331-348. MR0270219(42:5110)

Institut de Mathématiques de Bordeaux, CNRS UMR 5251, Equipe D'Analyse et Géométrie, Université Bordeaux 1, 351, Cours de la Libération, 33405 Talence, France E-mail address: Elmaati.Ouhabaz@math.u-bordeaux1.fr 